\title{
Heat transfer intensification during condensation of refrigerant with straight pipelines for a heat pump heating system
}

\author{
Sergey Fedosov ${ }^{1 *}$, Vadim Fedoseev ${ }^{2}$, and Svetlana Loginova ${ }^{2}$ \\ ${ }^{1}$ Moscow State Civil Engineering University, Moscow, 129337, Russia, \\ ${ }^{2}$ Ivanovo State Politechnical University, Ivanovo, 153000, Russia
}

\begin{abstract}
The subject of this study is to improve the design of the condenser for an air heat pump system in order to increase the heat exchange of the walls of the collectors (torus) and condenser pipelines with water for the heating circuit and to reduce the energy consumption for its operation. The emergence of the need to improve the existing models is due to the fact that the use in some cases of devices based on Venturi tubes and vortex tubes with reagent supply coaxially or tangentially does not give the desired positive effect. When their geometric parameters change, a decrease in the hydraulic resistance of the fluid and the volume of the working fluid leads to new configurations of the device. In our proposed device, the effect is achieved by reducing the total hydraulic resistance, increasing the efficiency of the heat pump system in a wide temperature range using condensers containing collectors (tori) and a container around the straight-pipe apparatus, where the straight-pipe apparatus together with the container represents a single structure of the "pipe in pipe".
\end{abstract}

\section{Introduction}

In the last decade, new energy-efficient technologies for life support of buildings, based on the use of heat pump systems for heat and cold supply (HPS), have become increasingly widespread in the world. In the most general configuration, heat pump systems of buildings or structures include the following main elements:

- Low-grade part - source of low-grade heat energy - low-grade heat collection system heat and cold supply system;

- High-potential part (consumer of thermal energy) - heating, ventilation and hot water supply systems of a building or structure.

The condenser is a heat exchanger that releases "useful" heat for the consumer, and the evaporator is a heat exchanger that utilizes (extracts) low-grade thermal energy: nontraditional renewable energy sources. Among the possible sources of low-grade thermal energy, it is necessary to highlight the following: soil, water, ambient air, etc.

The energy indicators of the heat transfer system of heat supply depend on climatic conditions, the type of low-potential heat sources, the temperature level of the heat supply

\footnotetext{
*Corresponding author: s179066171227@yandex.ru
} 
system, and the technical characteristics of the heat pump. The analysis of the efficiency of various sources of low-grade thermal energy shows that in the continental climatic conditions of Russia, the most economically promising are combined HPS of buildings and structures, using universally available ambient air as a low-potential heat source [1-5].

A heat pump condenser is a heat exchanger in which a high-potential heat source water - is heated by a condensing refrigerant. It is the main element of a heat pump and largely determines its technical and economic performance. Therefore, in order to increase the output thermal power of the air heat pump, and hence the efficiency (efficiency) of the heat pump system as a whole, it is necessary to increase its efficiency [6].

Technical losses in a heat exchanger (condenser) are losses caused by the presence of a finite temperature difference in them, which is associated with the finite dimensions of the heat exchange surfaces, the final values of the heat transfer coefficients, as well as hydraulic losses.

\section{Methods and Materials}

Condensers in heat pump systems are used to remove heat for heating communications for heating a room. In this case, the refrigerant in the condenser passes from a vapor state to a liquid state, i.e. condenses. In the thermodynamic cycle, this heat removed from the refrigerant during its operation is useful heat. Both liquids (water) and gases (air) can be used as cooling agents for condensers.

The composition and type of the heat-cooling substance (working fluid of the heating system), which receives the heat of condensation, depends on the method of application and the transfer of heat to the consumer. The use of the process of cooling the vaporous working fluid in the condenser and at the same time heating it, turning the body into a liquid state for the purposes of the consumer, is a function of transporting useful heat, which goes through the internal heating and water pipeline from the condenser to the place of its consumption, i.e. to the heating and hot water supply system.

Most often, condensers with a liquid-gas state are used, which, according to the design solution, are divided into shell-and-tube, two-tube (pipe in pipe), coil-pipe of special design.

Based on the materials of electronic libraries, the Federal Service for Intellectual Property (Rospatent), an effective model of a condenser related to the heat-refrigeration industry was developed, which can be used in heat pump systems. In 2019, a utility model patent No. 194145 was obtained [7].

This device works as follows. Through the pipeline (2), gaseous refrigerant enters the gas collector (1), where it is evenly distributed over the volume of the gas collector torus (1) and, with further movement, begins to condense, passing through the vertical pipelines (3). Then the cooled gaseous refrigerant enters the upper part of the subcooling manifold (4), where it completely condenses over the surface of the liquid refrigerant. Condensate fills half of the subcooling collector (4), then it is transported through cooling pipelines (5), after which the condensate enters the liquid refrigerant collector (6), made in the form of a parabolic container. After fulfilling its functions of heating the liquid (water) in the tank, through the pipeline (7), the liquid refrigerant is supplied to a three-way control valve (TRV) - a choke. This device is placed in a tank with liquid (water), where, due to heat exchange, the liquid that goes through the communications for heating will be heated (Fig. 1).

The use of this installation increases the surface of heat exchange of gaseous freon due to the walls of the condenser collector, which increases the transfer of heat to the water tank (8) and further to the heating circuit of the room. 
By using collectors in the form of tori, the system:

1. allows you to increase the heat exchange of the refrigerant with the heat circuit by increasing the area (volume) of heat exchange.

2. Allows you to evenly distribute the refrigerant on the walls of the collectors.

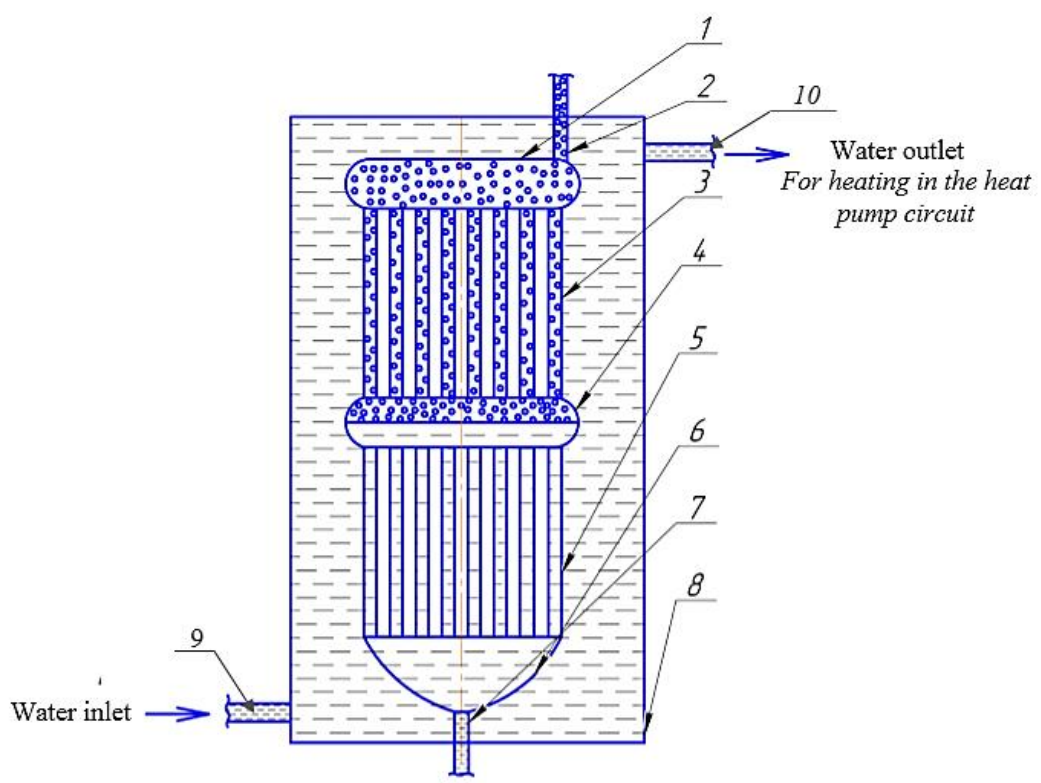

Fig. 1. Condenser for air heat pump system: (1) - gas collector; (2) - refrigerant pipeline; (3) condensing gas pipelines; (4) - collector of hypothermia; (5) - liquid refrigerant pipeline; (6) - a collector of liquid refrigerant; (7) - pipeline that leads the coolant to the throttle; (8) - water tank; (9) water inlet; 10 - water outlet

Let's compare the existing model of the coil type capacitor (Fig. 2) and the proposed one (Fig. 1). In a coil condenser, each coil, inside which there is a heating-water pipeline with an outlet to the heating system, contains additional hydraulic resistance due to bending. This movement wastes extra energy and time to travel through the pipeline.

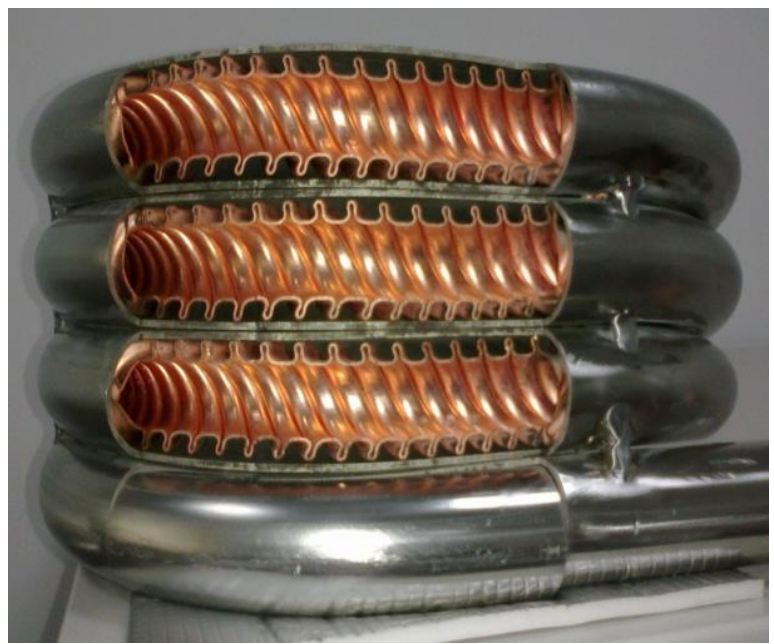

Fig. 2. Coil type capacitor model 
Analyzing this process, we will assume that a straight pipeline (Fig. 1) has a hydraulic resistance coefficient equal to $1[8,9]$, in a coiled pipeline the drag coefficient of one turn $\xi=0,5$ [9]. If the coil has 5 turns, then the maximum resistance coefficient $\xi_{\text {общ. }}$ will be 2.5 [9]. The standard wall thickness of the diameter of the condenser copper piping is $s=0,5 \mathrm{MM}$.

Let us show on the graph the dependence of the hydraulic resistance coefficient on a condenser with a coil geometry and in a condenser with a straight pipe (Fig. 3).

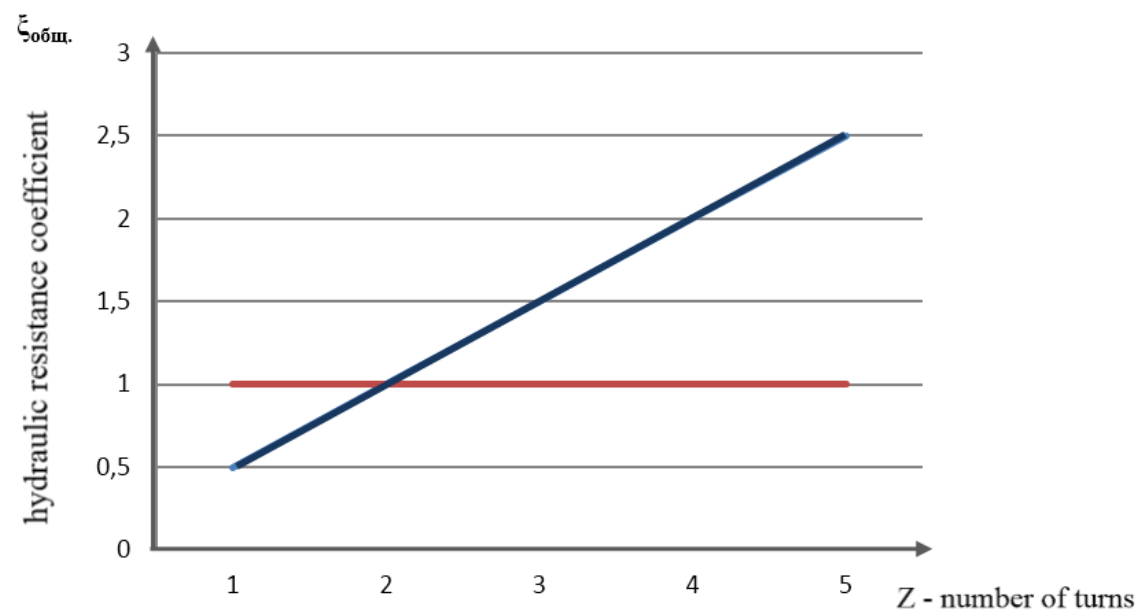

Fig. 3. Graph of dependences of the coefficient of hydraulic resistance

It can be seen from the graph that the increase in hydraulic resistance is significantly higher for a coil condenser, from which it follows that the power consumption of the compressor and the refrigerant transit time are higher than that of the condenser proposed by the authors with straight pipelines.

\section{Results}

It should be borne in mind that the increase in hydraulic resistance on the refrigerant side is determined only by the hydrostatic head, i.e. the height of the refrigerant column in the apparatus $\Delta p_{c m}^{\not \phi}$ [8]. The hydraulic resistance from the side of the coolant moving inside straight pipes consists of friction losses and local resistances.

The general equation for the hydraulic resistance to the flow of the coolant in a straighttube apparatus can be represented as the sum of these individual losses [8]:

$$
\begin{gathered}
\Delta p=\Delta p_{m \mathrm{p}}+\Delta p_{\text {ent }}+\Delta p_{\text {exit }} . \\
\Delta p=\left(\xi_{m p} \frac{L}{d_{\text {gu }}}+\zeta_{M .6 x}+1+\frac{\zeta_{M .6 x}+1}{z}\right) \cdot 0,5^{2} \cdot \rho,
\end{gathered}
$$

where: $\xi_{m p}$ - friction coefficient of coolant in straight pipes; $L$ - channel (pipe) length; $d_{B H}$ - inner pipe diameter; $\zeta_{M .6 x}=0,5$ - coefficient of local resistance; $Z$ - number of 
turns; $\rho$ - the density of the heat carrier; $\cdot \omega$ - the speed of the working medium in a given section.

When designing this type of condenser, it is necessary to take into account the hydraulic resistance of the coolant in the case of longitudinal flow around the entire bundle of straight pipes in the absence of transverse baffles, which are determined by the formula [8]:

$$
\Delta p=\Delta p_{\text {req. }}+\Delta p_{m}=\sum \xi_{\text {req. }} \cdot \frac{L}{d_{e}} \cdot \omega^{2} \cdot \frac{\rho}{2}+\sum \zeta_{m .} \cdot \omega^{2} \cdot \frac{\rho}{2}
$$

It should be noted that the hydrodynamic calculation of a condensation heat exchanger, which consists in determining the pressure losses $\Delta p$ caused by hydro- and aerodynamic resistances arising from the movement of working media (bodies) in a heat exchanger, will be indicated in the following works.

\section{Conclusions}

Methods for simply increasing the heat transfer surface area of the condenser in order to improve heat removal from the condenser device are exhausting [10-12]. The operation of such devices is based mainly on the transfer of heat due to the thermal conductivity of the working fluid. A device in the form of a casing filled with liquid (water) of the "pipe-inpipe" type has an advantage due to the convection of the liquid. The design proposed by us combines the advantages of the existing heat-cooling of the condenser with a decrease in hydraulic resistance, by eliminating unnecessary turns and bends, which significantly increases the reliability of the structure and this is clearly shown on the graph of the dependence of the coefficient of hydraulic resistance. During its development, this model will be quite suitable for replacing the existing condenser in the evaporative-condensing unit of the air heat pump [13].

\section{References}

1 S.V. Fedosov, V.N. Fedoseev, A.B. Petrukhin, L.A. Oparina, I.A. Martynov, Analysis of conditions in the design of energy-saving heat pump systems for autonomous textile production, Bulletin of the Volga State Technological University. Series: Materials. Constructions. Technology 2, $84-91$ (2018)

2 R.M. Aloyan, V.N. Fedoseev, I.A. Zaitseva, N.V. Vinogradova, Quantitative analysis of the configuration of the efficiency factor and thermal power of an air heat pump when heating low-rise textile buildings, Izvestiya vysshikh uchebnykh zavod. Textile industry technology 5 (371), 319-323 (2017)

3 R.M. Aloyan, V.N. Fedoseev, I.A. Zaitseva, N.V. Vinogradova, V.A. Emelin, V.A. Voronov, Comparative analysis of the combined modes of operation of the high-tech pump for low-rise buildings in the textile industry, Izvestia of higher educational institutions. Textile industry technology 5 (371), 324-328 (2017)

4 V.A. Voronov, V.A. Emelin, V.N. Fedoseev, I.A. Zaitseva, Climatic conditions and factors affecting the performance of an air heat pump, Theory and practice of technical, organizational, technological and economic solutions. Collection of scientific papers, 241-251 (2015) 
5 R.M. Aloyan, V.N. Fedoseev, S.M. Aloyan, I.A. Zaitseva, N.V. Vinogradova, Possible operating range of the air heat pump during the heating season, Proceedings of higher educational institutions. Textile Industry Technology 4 (370), 278-281 (2017)

6 S.V. Fedosov, V.N. Fedoseev, A.B. Petrukhin, Efficient energy saving of multi-storey buildings with an air heat pump, BST: Construction Technique Bulletin 2 (1026), 5053 (2020).

7 V.N. Fedoseev, S.V. Fedosov, P.I. Konyshev, L.A. Oparina, I.A. Sviridov, Patent for utility model RU 194145 U1, 29.11.2019. Capacitor. Application No.2019117153 dated 06.03.2019.

8 GN. Danilova, S.N. Bogdanov, O.P. Ivanov et al., Heat exchangers of refrigeration units (Mechanical engineering, L., 1986)

9 V.F. Gornov, M.N. Efremov, V.A. Leskov, N.V. Shilkin, Heat pump systems for cottages, Energy Saving 1, 30-37 (2019)

10 Yu.A. Tabunshchikov, M.M. Brodach, Energy efficient buildings and innovative engineering systems, AVOK: Ventilation, heating, air conditioning, heat supply and building thermal physics 1, 6-11 (2014)

11 Yu.A. Tabunshchikov, M.M. Brodach, System analysis of the design of energy efficient buildings, Architecture and modern information technology 8, 14 (2015)

12 M.M. Brodach, N.V. Shilkin, Low-rise multi-apartment residential buildings as a new habitat, Energy saving 5, 1-7 (2020)

13 V.N. Fedoseev, V.A. Emelin, V.A. Voronov, Yu.E. Ostryakova, I.A. Sviridov, RF patent 174083. Heat pump. Appl. dated 09.01.2017. Publ. 09.29.2017. Bul. No. 28. 